\title{
NON-ZEEMAN CIRCULAR POLARIZATION OF MOLECULAR ROTATIONAL SPECTRAL LINES
}

\author{
Martin Houde $^{1,2}$, Talayeh Hezareh $^{3}$, Scott Jones $^{1}$, and Fereshte Rajabi ${ }^{1}$ \\ ${ }^{1}$ Department of Physics and Astronomy, The University of Western Ontario, London, ON, N6A 3K7, Canada \\ 2 Division of Physics, Mathematics and Astronomy, California Institute of Technology, Pasadena, CA 91125, USA \\ ${ }^{3}$ Max-Planck-Institut für Radioastronomie, Auf dem Hügel 69, D-53121 Bonn, Germany \\ Received 2012 August 19; accepted 2012 December 10; published 2013 January 21
}

\begin{abstract}
We present measurements of circular polarization from rotational spectral lines of molecular species in Orion $\mathrm{KL}$, most notably ${ }^{12} \mathrm{CO}(J=2 \rightarrow 1)$, obtained at the Caltech Submillimeter Observatory with the FourStokes-Parameter Spectral Line Polarimeter. We find levels of polarization of up to $1 \%-2 \%$ in general; for ${ }^{12} \mathrm{CO}(J=2 \rightarrow 1)$ this level is comparable to that of linear polarization also measured for that line. We present a physical model based on resonant scattering in an attempt to explain our observations. We discuss how slight differences in scattering amplitudes for radiation polarized parallel and perpendicular to the ambient magnetic field, responsible for the alignment of the scattering molecules, can lead to the observed circular polarization. We also show that the effect is proportional to the square of the magnitude of the plane of the sky component of the magnetic field and therefore opens up the possibility of measuring this parameter from circular polarization measurements of Zeeman insensitive molecules.
\end{abstract}

Key words: ISM: clouds - ISM: individual objects (Orion KL) - ISM: magnetic fields - ISM: molecules polarization

\section{INTRODUCTION}

Magnetic field studies in molecular clouds are most often conducted through the detection of polarization signals, in either molecular lines or dust continuum spectra. Except for Zeeman measurements on suitable molecular species, which directly probe the strength of the magnetic field (usually the line-ofsight component; see Heiles 1997; Crutcher et al. 1999; Brogan \& Troland 2001; Falgarone et al. 2008), all other types of observations and analyses provide indirect characterizations of magnetic fields. Perhaps the only technique that does not rely on polarization measurements is that of Houde et al. (2000a) and Li \& Houde (2008), which is based on the comparison of line widths from coexistent molecular ion and neutral species (see also Houde et al. 2000b, 2001; Tilley \& Balsara 2010, 2011; Falceta et al. 2010).

For a couple of decades or so measurements of dust continuum polarization, in either emission or absorption (Heiles 2000; Novak et al. 2004; Li et al. 2006; Matthews et al. 2009; Dotson et al. 2010; Vaillancourt \& Matthews 2012), have been a particularly efficient way of conducting systematic studies on the interstellar medium and the effects and role of magnetic fields in the processes leading to the formation of stars (Girart et al. 2006; Attard et al. 2009). Although it has been known since the work of Chandrasekhar \& Fermi (1953) that polarization maps can be used to provide an estimate of the strength of the plane of the sky component of the magnetic field, recent developments have rendered possible refinements of this technique and the further characterization of magnetized turbulence and its power spectrum (Houde 2004; Heyer et al. 2008; Hildebrand et al. 2009; Houde et al. 2009, 2011; Chitsazzadeh et al. 2012).

Linear polarization of molecular lines through the so-called Goldreich-Kylafis effect (Goldreich \& Kylafis 1981), which was first confirmed observationally in the envelope of an evolved star some 16 years after its theoretical prediction (Glenn et al. 1997), is now routinely detected in star-forming regions. This effect specifies the conditions leading to an imbalance in the population of magnetic sub-levels responsible for the $\pi$ - and $\sigma$-transitions, which are respectively polarized parallel and perpendicular to the orientation of the plane of the sky component of the magnetic field. Depending on which sublevel populations dominate, the detected linear polarization can be oriented in either direction. It is in principle possible to lift this degeneracy through the observations of several molecular transitions (Cortes et al. 2005). With the development of array heterodyne receivers it is to be expected that this technique will also become an important tool for mapping magnetic fields on large scales in the interstellar medium.

The prediction and observations of circular polarization signals, beyond the measurement of the Zeeman effect, have proven even more challenging. But improvements are happening on the observational front. Recent observations by Muñoz et al. (2012) have successfully revealed the detection of continuum circular polarization levels of about $1 \%$ at wavelengths of $1.3 \mathrm{~mm}$ and $860 \mu \mathrm{m}$ in Sgr A*. They attribute the presence of such signals to a conversion of linear polarization to circular polarization (or Faraday conversion). For molecular lines, Cotton et al. (2011) have reported the clear detection of non-Zeeman circular polarization of $\mathrm{SiO}$ maser lines in the asymptotic branch giant star IK Tau with the Very Long Baseline Array. They found high levels of circular polarization (often exceeding 10\%) that can also amount to a significant fraction of the linear polarization simultaneously detected. They were unable to explain their observations using alternate models for the conversion of linear polarization to circular polarization based on population imbalance (e.g., Wiebe \& Watson 1998; Deguchi \& Watson 1985). It thus appears that a novel physical model is needed to explain the presence of such polarization signals in molecular lines.

This is what we endeavor to accomplish in this paper, where we present the recent detection of circular polarization signals in rotational spectral lines of molecular species in Orion KL, most notably ${ }^{12} \mathrm{CO}(J=2 \rightarrow 1)$ (Sections 2 and 3$)$. We also introduce a physical model based on resonant scattering in an attempt to explain our observations (Section 4). We follow with a discussion in Section 5 and end with a short summary in Section 6. 


\section{OBSERVATIONS}

The observations discussed in this paper were obtained at the Caltech Submillimeter Observatory (CSO) with the FourStokes-Parameter Spectral Line Polarimeter (FSPPol; Hezareh $\&$ Houde 2010). FSPPol allows measurements of linear and circular polarization signals by the insertion of half-wave (HWP) and quarter-wave (QWP) plates in the CSO telescope beam before detection using the facility's receivers, which are sensitive to a single state of linear polarization. Although FSPPol can operate in the $230 \mathrm{GHz}, 345 \mathrm{GHz}$, and $492 \mathrm{GHz}$ bands with the corresponding receivers, the observations presented in this paper were all conducted in the $230 \mathrm{GHz}$ band. More details on the functioning of FSPPol can be found in Hezareh \& Houde (2010).

The circular polarization observations of ${ }^{12} \mathrm{CO}(J=2 \rightarrow 1)$ at $230.5 \mathrm{GHz}$ presented and discussed in this paper were obtained on 2011 November 23 under mediocre conditions $(\tau(225 \mathrm{GHz}) \approx 0.2)$ and on 2012 February 5 under excellent skies $(\tau(225 \mathrm{GHz}) \approx 0.03-0.04)$. Both sets of observations yielded similar results; the spectrum stemming from the 2012 February observations is shown in Figure 1. Also discussed are subsequent circular polarization measurements aimed at the $\operatorname{HCN}(J=3 \rightarrow 2)$ rotational transition at $265.9 \mathrm{GHz}$, which were realized on 2012 February 8 and $9(\tau(225 \mathrm{GHz}) \approx$ $0.05)$ and shown in Figure 2. Finally, linear polarization of ${ }^{12} \mathrm{CO}(J=2 \rightarrow 1)$ observations obtained on 2012 February 12 $(\tau(225 \mathrm{GHz}) \approx 0.13)$ is shown in Figure 3. This linear polarization spectrum is in good agreement with the previous result of Girart et al. (2004; see the two central panels of their Figure 1). All observations were pointed to the peak position of Orion KL at R.A. $(\mathrm{J} 2000)=05^{\mathrm{h}} 35^{\mathrm{m}} 14.5$ and Decl. (J2000) $=-05^{\circ} 22^{\prime} 30^{\prime} \cdot 4$. The telescope efficiency was measured to be $\approx 60 \%$ from scans on Jupiter, while the pointing accuracy was determined to be better than approximately $6^{\prime \prime}$.

In order to minimize instrumental effects in the detection of polarization signals due to pointing and calibration errors, a conservative observation method was adopted. That is, the necessary integrations for the measurement of linear polarization (at four HWP orientations) and circular polarization (at two QWP orientations) were kept short at one minute ON-source, and a temperature calibration was done before each of them (see Hezareh \& Houde 2010). The low levels of polarization at the peaks of strong lines (i.e., on the order of, or a few times, $0.1 \%$ for ${ }^{12} \mathrm{CO}$ and $\mathrm{HCN}$ ) found in all these spectra are an indication of the benefits in using this method and can be taken as an approximation for the level of instrument polarization present in these observations.

\section{RESULTS}

Our measurement of circular polarization for ${ }^{12} \mathrm{CO}(J=$ $2 \rightarrow 1$ ) in Orion KL reveals a clear detection of polarization levels of up to approximately $1 \%-2 \%$ across the spectral line, as can be seen in Figure 1. Shown are the Stokes $I$ spectrum, uncorrected for telescope efficiency, and polarization levels (symbols with uncertainty, using the scale on the right) in the top panel, while the Stokes $V$ spectrum is displayed in the bottom panel. All polarization data satisfy $p \geqslant 3 \sigma_{p}$, where $p$ and $\sigma_{p}$ are the polarization level and its uncertainty, respectively. These relatively low polarization levels are still significantly higher than the instrumentation polarization expected with FSPPol. For example, preliminary measurements of the Zeeman effect on $\mathrm{CN}(N=2 \rightarrow 1)$ presented in Hezareh \& Houde (2010)

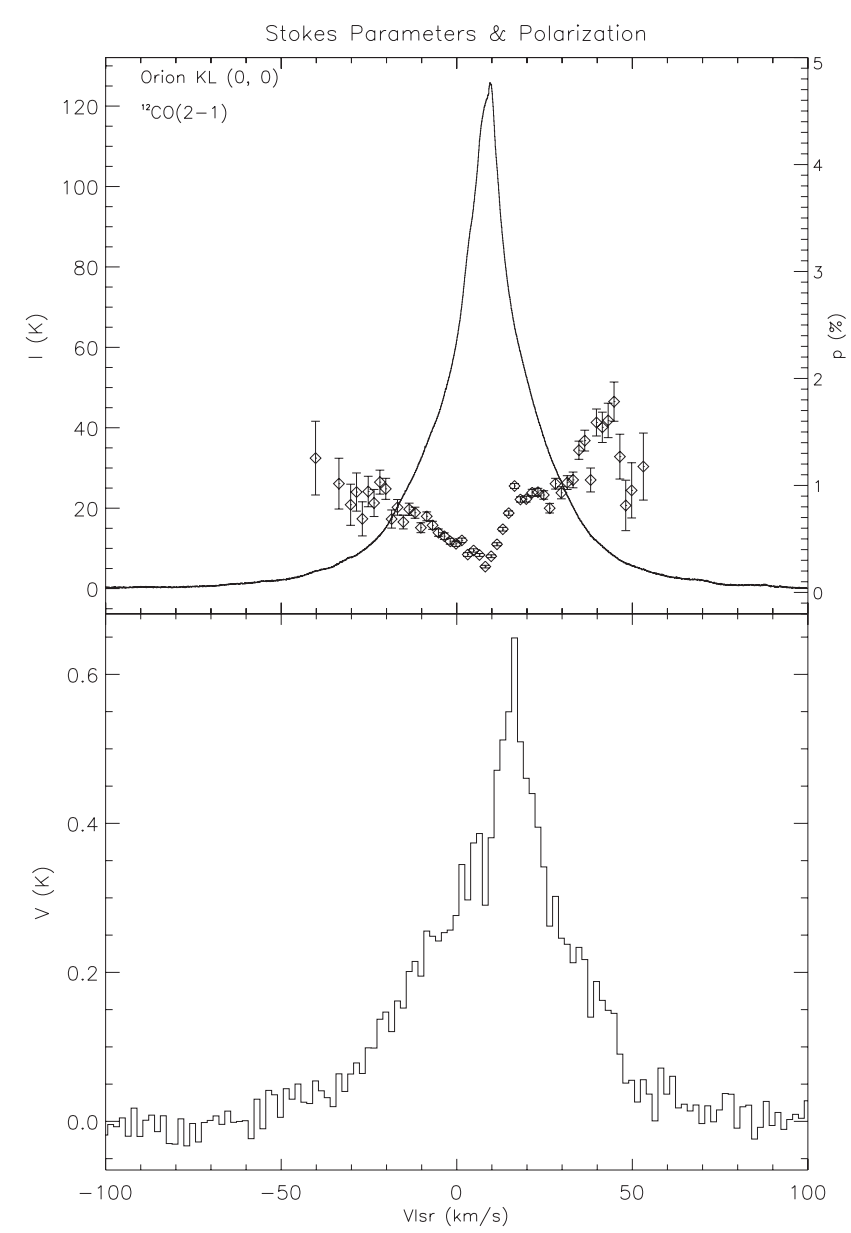

Figure 1. Circular polarization spectrum of the ${ }^{12} \mathrm{CO}(J=2 \rightarrow 1)$ observations made at the peak position of Orion KL (R.A. (J2000) $=05^{\mathrm{h}} 35^{\mathrm{m}} 14.5$, Decl. (J2000) $\left.=-05^{\circ} 22^{\prime} 30^{\prime \prime} 4\right)$ on 2012 February 5 at the CSO with FSPPol. Top: Stokes $I$ spectrum, uncorrected for telescope efficiency, and circular polarization levels (symbols with uncertainty, using the scale on the right). All polarization data satisfy $p \geqslant 3 \sigma_{p}$, where $p$ and $\sigma_{p}$ are the polarization level and its uncertainty, respectively. Bottom: the Stokes $V$ spectrum, also uncorrected for telescope efficiency. The frequency resolution of the Stokes $I$ spectrum is $61 \mathrm{kHz}$ $\left(0.08 \mathrm{~km} \mathrm{~s}^{-1}\right)$, while the Stokes $V$ spectrum was smoothed by a factor of 20 .

show that less than $0.2 \%$ of Stokes $I$ is expected to leak into Stokes $V$. This is consistent with the polarization level detected at the peak of our ${ }^{12} \mathrm{CO}(J=2 \rightarrow 1)$ Stokes $V$ spectrum (and HCN $(J=3 \rightarrow 2)$; see below), which should be approximately zero in view of the very high optical depth expected for this line. Although the probability of getting false Stokes $V$ Zeeman profiles (i.e., in the shape of the velocity derivative of Stokes $I$ ) is non-negligible with such observations, our spectrum shows no such obvious pattern.

Nonetheless, steps were taken to ensure that this detection is not spurious. First, the circular polarization of ${ }^{12} \mathrm{CO}(\mathrm{J}=$ $2 \rightarrow 1)$ was measured twice, once in 2011 November and again in 2012 February, under different sky conditions with similar results. Second, we performed independent observations aimed at the $\mathrm{HCN}(J=3 \rightarrow 2)$ transition at the same position, which is also known to be strong in Orion KL (Houde et al. 2000a) $;{ }^{4}$ the result from these observations is shown in the left

\footnotetext{
4 Although the frequency of that line is different from the design frequency of the QWP (i.e., $\approx 266 \mathrm{GHz}$ vs. $226 \mathrm{GHz}$ ), it can be shown that the only effect caused by the error in the thickness of the QWP at a given frequency is a reduction in sensitivity to incoming circular polarization proportional to the cosine of the thickness error. That is, no incident linear polarization signal can be converted into circular polarization with the FSPPol setup.
} 

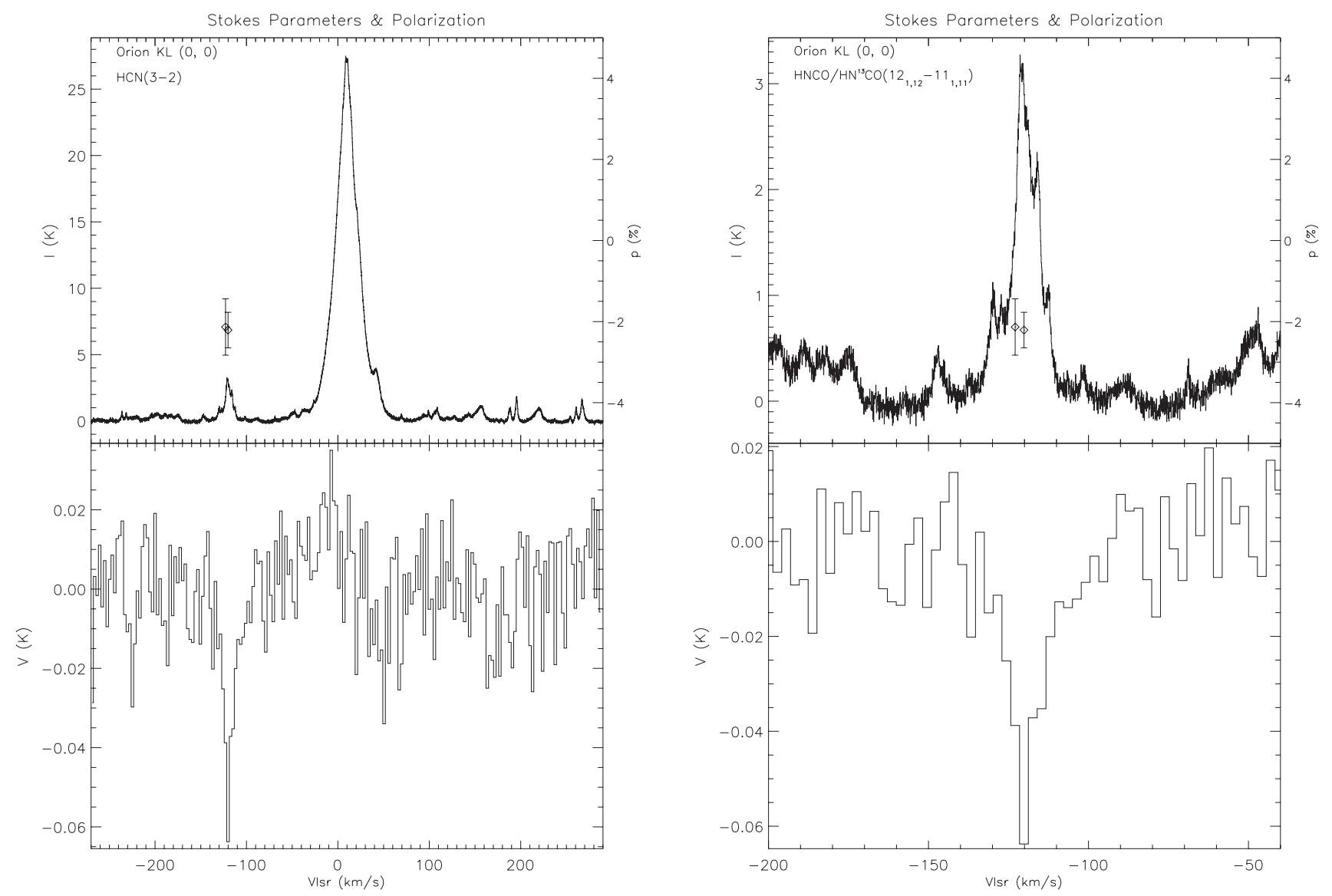

Figure 2. Left: same as Figure 1 but for $\operatorname{HCN}(J=3 \rightarrow 2)$, obtained on 2012 February 8 and 9 . The spectral feature located at $\approx-120 \mathrm{~km} \mathrm{~s}^{-1}$ is a blend of lines from a few molecular species, most notably HNCO and $\mathrm{HN}^{13} \mathrm{CO}$ in the $\left(N_{K_{a} K_{c}}=12_{1,12} \rightarrow 11_{1,11}\right)$ transitions; a close-up of this spectrum is shown in the right panel. The frequency resolution of the Stokes $I$ spectra is $61 \mathrm{kHz}\left(0.07 \mathrm{~km} \mathrm{~s}^{-1}\right)$, while the Stokes $V$ spectra were smoothed by a factor of 40 .

panel of Figure 2. As can be seen from the figure, there is no detection of circular polarization up to a level of approximately $0.1 \%$ in $\mathrm{HCN}(J=3 \rightarrow 2)$, which is an order of magnitude less than the ${ }^{12} \mathrm{CO}(J=2 \rightarrow 1)$ detection of Figure 1. But most interestingly, we see the presence of circular polarization at a level of approximately $-2 \%$ at the peak of a spectral feature located at $\approx-120 \mathrm{~km} \mathrm{~s}^{-1}$, which is mostly dominated by the $N_{K_{a} K_{c}}=12_{1,12} \rightarrow 11_{1,11}$ transitions of $\mathrm{HNCO}$ and $\mathrm{HN}^{13} \mathrm{CO}$ in the image band at $262.8 \mathrm{GHz}$; a close-up of this spectrum is shown in the right panel of the same figure. The fact that simultaneous measurements on two spectral features (i.e., $\mathrm{HCN}$ and $\mathrm{HNCO} / \mathrm{HN}^{13} \mathrm{CO}$ ) yield none and a detection is strong evidence that our observations of ${ }^{12} \mathrm{CO}(J=2 \rightarrow 1)$ are not spurious but result from true circular polarization signals present in the spectral line. In the next section, we present a physical model that seeks to explain these observations.

\section{ANALYSIS}

We consider a molecule immersed in a medium that harbors a magnetic field, which provides spatial alignment. We further assume the existence of incident radiation close to, or at, a resonant frequency of the molecule and in a state of linear polarization at some angle relative to the orientation of the magnetic field. We can imagine a situation where a population of a given molecular species in a background medium emits radiation that is linearly polarized, for example, through the Goldreich-Kylafis effect, which is then incident on similar molecules located in the foreground where the orientation of the magnetic field has changed.
We will investigate the conditions necessary to transform linear polarization into circular polarization. Such a situation has been previously considered in the literature (e.g., Deguchi \& Watson 1985), but the mechanism for the generation of circular polarization presented below is different.

\subsection{Conversion of Linear to Circular Polarization}

We denote this incident background radiation state by $\left|\psi_{0}\right\rangle$, which is linearly polarized at an angle $\theta$ with the foreground magnetic field, which we assume to be located in the plane normal to the direction of propagation, for simplicity (this assumption will be dropped later on). This radiation state can be decomposed as follows:

$$
\left|\psi_{0}\right\rangle=\alpha_{0}\left|n_{\|}\right\rangle+\beta_{0}\left|n_{\perp}\right\rangle
$$

where $\alpha_{0}=\cos (\theta)$ and $\beta_{0}=\sin (\theta)$, while $\left|n_{\|}\right\rangle$and $\left|n_{\perp}\right\rangle$ are $n$-photon states linearly polarized parallel and perpendicular to the foreground magnetic field, respectively, propagating toward the observer. These states are orthogonal to one another and normalized. The basic idea is to determine whether these two states scatter differently off a foreground molecule in such a manner that a small relative phase shift $\phi^{\prime}$ is introduced between them. The scattered radiation state would then become (up to a global phase term)

$$
\begin{aligned}
\left|\psi^{\prime}\right\rangle & \simeq \alpha_{0}\left(1+i \phi^{\prime}\right)\left|n_{\|}\right\rangle+\beta_{0}\left|n_{\perp}\right\rangle \\
& \simeq \alpha_{0} e^{i \phi^{\prime}}\left|n_{\|}\right\rangle+\beta_{0}\left|n_{\perp}\right\rangle,
\end{aligned}
$$




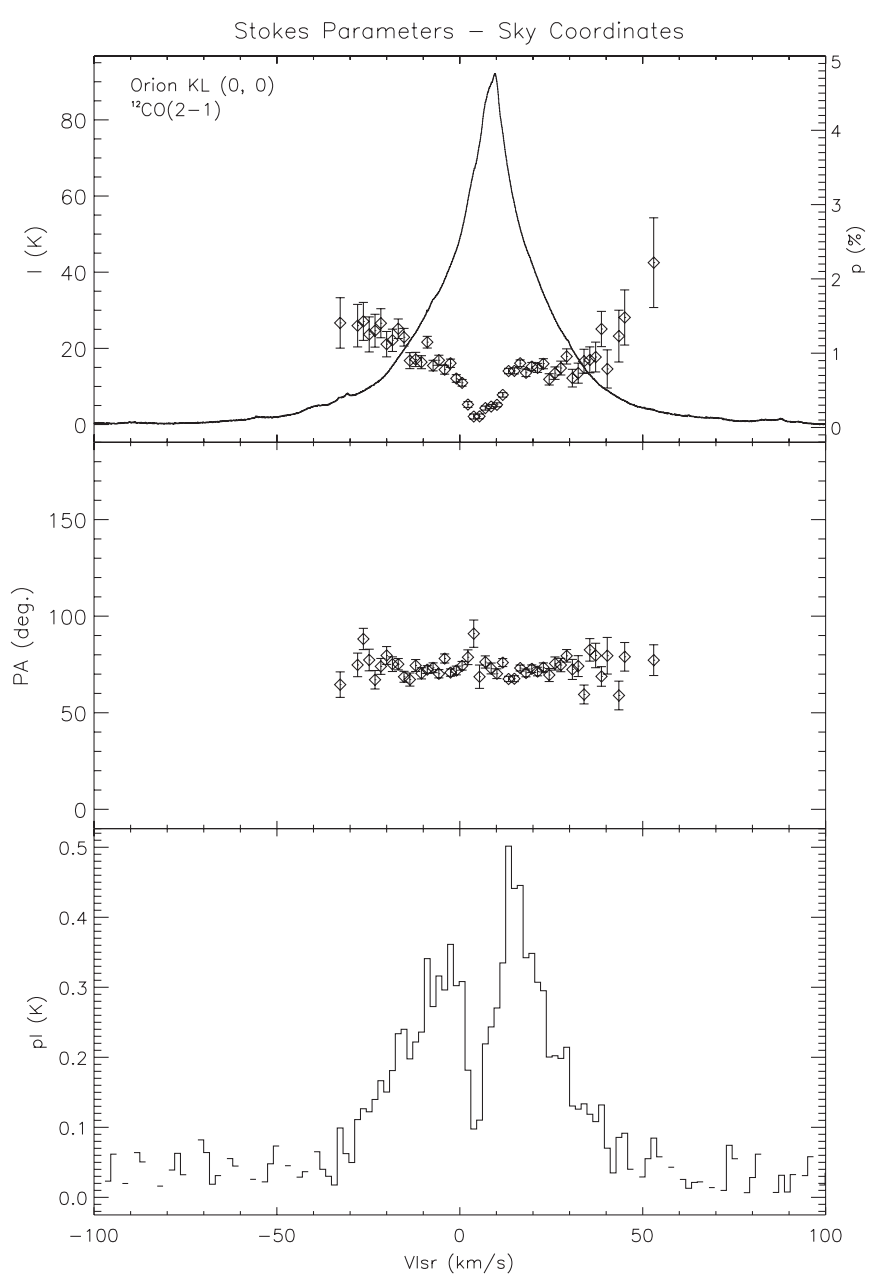

Figure 3. Linear polarization spectrum of the ${ }^{12} \mathrm{CO}(J=2 \rightarrow 1)$ observations made at the peak position of Orion KL (R.A. (J2000) $=05^{\mathrm{h}} 35^{\mathrm{m}} 14.5$, Decl. (J2000) $\left.=-05^{\circ} 22^{\prime} 30^{\prime \prime} 4\right)$ on 2012 February 12 at the CSO with FSPPol. Top: Stokes $I$ spectrum, uncorrected for telescope efficiency, and linear polarization levels (symbols with uncertainty, using the scale on the right). All polarization data satisfy $p \geqslant 3 \sigma_{p}$, where $p$ and $\sigma_{p}$ are the polarization level and its uncertainty, respectively, and are corrected for positive bias in the polarized flux. Middle: polarization angle from north, increasing eastward. Bottom: polarized flux $p I$, also uncorrected for telescope efficiency, but corrected for positive bias due to noise. The frequency resolution of the Stokes $I$ spectrum is $61 \mathrm{kHz}\left(0.08 \mathrm{~km} \mathrm{~s}^{-1}\right)$, while the polarized spectrum was smoothed by a factor of 20 .

where it was assumed that the separate phase shifts for both states are much less than unity (therefore $\phi^{\prime} \ll 1$ follows for the last equation). It is expected, however, that a very large number of scattering $N$ will occur for a given radiation state as it propagates within a molecular cloud. Each scattering will contribute a relative phase shift $\phi^{\prime}$, and the final radiation state becomes

$$
|\psi\rangle \simeq \alpha_{0} e^{i \phi}\left|n_{\|}\right\rangle+\beta_{0}\left|n_{\perp}\right\rangle
$$

with $\phi=N \phi^{\prime}$.

We can also define $n$-photon circular polarization states with

$$
\left|n_{ \pm}\right\rangle=\frac{1}{\sqrt{2}}\left(\left|n_{\|}\right\rangle \pm i\left|n_{\perp}\right\rangle\right) .
$$

It is easy to show that the level of circular polarization (Stokes) $v$ for the final state is

$$
\begin{aligned}
v & =\left\|\left\langle n_{+} \mid \psi\right\rangle\right\|^{2}-\left\|\left\langle n_{-} \mid \psi\right\rangle\right\|^{2} \\
& =-2 \alpha_{0} \beta_{0} \sin (\phi) .
\end{aligned}
$$

We can also define the complete state of linear polarization by introducing complementary states of linear polarization oriented at $\pm 45^{\circ}$ from the magnetic field direction

$$
\left|n_{ \pm 45}\right\rangle=\frac{1}{\sqrt{2}}\left(\left|n_{\|}\right\rangle \pm\left|n_{\perp}\right\rangle\right)
$$

and the (Stokes) parameters $q$ and $u$ with

$$
\begin{gathered}
q=\left\|\left\langle n_{\|} \mid \psi\right\rangle\right\|^{2}-\left\|\left\langle n_{\perp} \mid \psi\right\rangle\right\|^{2} \\
=\alpha_{0}^{2}-\beta_{0}^{2} \\
u=\left\|\left\langle n_{+45} \mid \psi\right\rangle\right\|^{2}-\left\|\left\langle n_{-45} \mid \psi\right\rangle\right\|^{2} \\
=2 \alpha_{0} \beta_{0} \cos (\phi) .
\end{gathered}
$$

These can be compared with corresponding parameters for the incident radiation state

$$
\begin{gathered}
q_{0}=\alpha_{0}^{2}-\beta_{0}^{2} \\
u_{0}=2 \alpha_{0} \beta_{0} \\
v_{0}=0
\end{gathered}
$$

to find that, for the chosen system of reference, linear polarization is being converted from $u_{0}$ to circular polarization $v$, and that the total amount of polarization is conserved.

The previous example considered the case of strictly forward scattering where the radiation states before and after scattering were composed of the same $\left|n_{\|}\right\rangle$and $\left|n_{\perp}\right\rangle$ states. We can generalize the analysis by considering a number of initial states $\left|\psi_{j}\right\rangle$ propagating in different directions and/or containing different numbers of photons (but of the same frequency) such that

$$
\left|\psi_{j}\right\rangle=\alpha_{j}\left|n_{j, \|}\right\rangle+\beta_{j}\left|n_{j, \perp}\right\rangle .
$$

These states could all potentially scatter in the direction of the observer into $\left|n_{\|}\right\rangle$and $\left|n_{\perp}\right\rangle$. The incident radiation state then becomes

$$
|\psi\rangle=\sum_{j}\left(\alpha_{j}\left|n_{j, \|}\right\rangle+\beta_{j}\left|n_{j, \perp}\right\rangle\right),
$$

with $\sum_{j}\left(\alpha_{j}^{2}+\beta_{j}^{2}\right)=1$. The scattered state, ascribed to $j=0$ (i.e., $\left|n_{0, \|}\right\rangle=\left|n_{\|}\right\rangle$and $\left|n_{0, \perp}\right\rangle=\left|n_{\perp}\right\rangle$ ), retains the same form as Equation (2) (after normalization),

$$
\left|\psi^{\prime}\right\rangle \simeq \alpha^{\prime} e^{i \phi^{\prime}}\left|n_{\|}\right\rangle+\beta^{\prime}\left|n_{\perp}\right\rangle
$$

when

$$
\begin{gathered}
\alpha^{\prime}=B C \alpha_{0} \\
\beta^{\prime}=C \beta_{0}
\end{gathered}
$$

$$
\begin{aligned}
\phi^{\prime}= & \frac{1}{D}\left[\operatorname{Re}\left\{\sum_{j} \phi_{j, \|}^{\prime} \frac{\alpha_{j}}{\alpha_{0}}\right\}\left(1-\operatorname{Im}\left\{\sum_{k} \phi_{k, \perp}^{\prime} \frac{\beta_{k}}{\beta_{0}}\right\}\right)\right. \\
& \left.-\operatorname{Re}\left\{\sum_{j} \phi_{k, \perp}^{\prime} \frac{\beta_{j}}{\beta_{0}}\right\}\left(1-\operatorname{Im}\left\{\sum_{k} \phi_{k, \|}^{\prime} \frac{\alpha_{j}}{\alpha_{0}}\right\}\right)\right],
\end{aligned}
$$


where

$$
\begin{aligned}
& B= \frac{1}{\left\|1+i \sum_{k} \phi_{k, \perp}^{\prime} \frac{\beta_{k}}{\beta_{0}}\right\|^{2}}\left[1-\sum_{j}\left(\operatorname{Im}\left\{\phi_{j, \|}^{\prime} \frac{\alpha_{j}}{\alpha_{0}}\right\}\right.\right. \\
&\left.+\operatorname{Im}\left\{\phi_{j, \perp}^{\prime} \frac{\beta_{j}}{\beta_{0}}\right\}\right)+\sum_{j, k}\left(\operatorname{Re}\left\{\phi_{j, \|}^{\prime} \frac{\alpha_{j}}{\alpha_{0}}\right\} \operatorname{Re}\left\{\phi_{k, \perp}^{\prime} \frac{\beta_{k}}{\beta_{0}}\right\}\right. \\
&\left.\left.+\operatorname{Im}\left\{\phi_{j, \|}^{\prime} \frac{\alpha_{j}}{\alpha_{0}}\right\} \operatorname{Im}\left\{\phi_{k, \perp}^{\prime} \frac{\beta_{k}}{\beta_{0}}\right\}\right)\right] \\
& C= \frac{1+i \sum_{k} \phi_{k, \perp}^{\prime} \frac{\beta_{k}}{\beta_{0}}}{\sqrt{\alpha_{0}^{2}\left\|1+i \sum_{k} \phi_{k, \|}^{\prime}\right\| \frac{\alpha_{k}}{\alpha_{0}}\left\|^{2}+\beta_{0}^{2}\right\| 1+i \sum_{k} \phi_{k, \perp}^{\prime} \frac{\beta_{k}}{\beta_{0}} \|^{2}}} \\
& D=B\left\|1+i \sum_{k} \phi_{k, \perp}^{\prime} \frac{\beta_{k}}{\beta_{0}}\right\|^{2}
\end{aligned}
$$

and $\operatorname{Re}\{\cdots\}$ and $\operatorname{Im}\{\cdots\}$ stand for the real and imaginary parts, respectively. In Equations (15)-(20) $\phi_{j, \|}^{\prime}$ and $\phi_{j, \perp}^{\prime}$ are the scattering amplitudes for the different polarization states, while $\alpha_{j}$ and $\beta_{j}$ are assumed complex in general (except for $\alpha_{0}$ and $\beta_{0}$, which are chosen to be real). The scattering amplitudes $\phi_{j, \|}^{\prime}$ and $\phi_{j, \perp}^{\prime}$ will contain geometrical factors that will account for the different incidence angles. It can be verified that the earlier forward scattering case is recovered (up to a global phase term) when $j=k=0$ is the only possibility, the imaginary components are zero, and $\phi_{\|}^{\prime}, \phi_{\perp}^{\prime} \ll 1$. The form of Equation (3) and the others that follow are therefore still adequate for the more general case.

\subsection{Dielectric Susceptibility}

The approach taken in the previous section may appear counterintuitive with respect to more common types of analyses encountered when dealing with molecular polarizability. More precisely, it could seem more natural to investigate the relative phase shift between the scattered and incident radiations, due to the interaction with a molecule, through calculations of the induced molecular dielectric susceptibility (Grynberg et al. 2010; Cohen-Tannoudji et al. 1988, 1977). We will show here that this approach cannot account for the effect we will discuss in this paper.

For a detailed and more realistic treatment of radiation/molecule interactions involving two molecular energy levels it is often preferable to use the density operator (or density matrix) $\hat{\sigma}$ to perform the analysis. Under this formalism it is found that the $j$ th component of the mean electric dipole moment $\hat{d}_{j}$ can be evaluated with

$$
\left\langle\hat{d}_{j}\right\rangle=\operatorname{Tr}\left\{\hat{\sigma} \hat{d}_{j}\right\}=\sum_{a, b} \sigma_{b a} d_{j, a b},
$$

where $\operatorname{Tr}\{\cdots\}$ denotes the trace and $\sigma_{b a}=\langle b|\hat{\sigma}| a\rangle$ is a matrix element, with $|b\rangle$ the quantum state for level $b$, etc. With the knowledge of $\left\langle\hat{d}_{j}\right\rangle$ one can determine the induced molecular polarization component $P_{j}=\left\langle\hat{d}_{j}\right\rangle / V$, with $V$ the volume under consideration, and the dielectric susceptibility $\chi_{j}$ through

$$
P_{j}=\chi_{j} E_{0, j}
$$

with $\mathbf{E}_{0}$ the incident electric field.
By performing these calculations for each linear polarization component, one can determine the relative phase shift induced between the two scattered electric field components from the difference in the corresponding dielectric susceptibilities. For example, if we respectively have $\chi_{\|}$and $\chi_{\perp}$ for the susceptibilities parallel and perpendicular to the orientation of the magnetic field responsible for the alignment of the molecule, then the relative phase shift $\varphi$ is given by

$$
\varphi \simeq\left(\chi_{\|}-\chi_{\perp}\right) \frac{\omega}{2 c} \Delta L,
$$

with $\omega, c$, and $\Delta L$ the frequency of radiation, the speed of light, and the propagation path length after scattering, respectively. Such calculations, when applied to the case treated in this paper, reveal that $\varphi$ is several orders of magnitude too small to explain our observations.

It is important to note that because selection rules for electric dipole transitions specify that $d_{j, a b}=0$ when $a=b$, the only components of the density matrix involved in these calculations are off-diagonal elements, i.e., $\sigma_{b a}$ for $b \neq a$ (see Equation (21)). As we will see in the following section, the resonant scattering process we study is of the second order in the electric field and only appears in the diagonal elements of the density matrix. More precisely, if an expansion of the density matrix in terms of powers of the interaction Hamiltonian $\hat{H}_{\mathrm{I}}$ is used (Grynberg et al. 2010), then it can be shown that the second-order diagonal terms are

$$
\begin{aligned}
\sigma_{a a}^{(2)}= & -\frac{1}{\hbar^{2}} \sum_{b \neq a}\left(\sigma_{a a}^{(0)}-\sigma_{b b}^{(0)}\right) \int_{t_{0}}^{t} e^{-\Gamma_{a}\left(t-t^{\prime}\right)} \\
& \times\left[\left\langle a\left|H_{\mathrm{I}}\left(t^{\prime}\right)\right| b\right\rangle \int_{t_{0}}^{t^{\prime}}\left\langle b\left|H_{\mathrm{I}}\left(t^{\prime \prime}\right)\right| a\right\rangle e^{-\left(i \omega_{b a}+\gamma_{b a}\right)\left(t^{\prime}-t^{\prime \prime}\right)} d t^{\prime \prime}\right. \\
& \left.+\left\langle b\left|H_{\mathrm{I}}\left(t^{\prime}\right)\right| a\right\rangle \int_{t_{0}}^{t^{\prime}}\left\langle a\left|H_{\mathrm{I}}\left(t^{\prime \prime}\right)\right| b\right\rangle e^{-\left(i \omega_{b a}+\gamma_{b a}\right)\left(t^{\prime}-t^{\prime \prime}\right)} d t^{\prime \prime}\right] d t^{\prime} .
\end{aligned}
$$

In Equation (24) $\Gamma_{a}$ is the relaxation rate of level $a, \omega_{b a}$ and $\gamma_{b a}$ are, respectively, the frequency and relaxation coefficient for a transition between states $|b\rangle$ and $|a\rangle$, and $t-t_{0}$ is the duration of the interaction. Since the interaction Hamiltonian involves the electric dipole moment (i.e., $\left.\hat{H}_{\mathrm{I}}=-\hat{\mathbf{d}} \cdot \mathbf{E}\right)$, it brings a scattering of radiation where the molecule initially in state $|a\rangle$ is momentarily excited to (the virtual) state $|b\rangle$ before settling back to $|a\rangle$. As was mentioned earlier, this process cannot be captured in calculations involving dielectric susceptibilities described through Equations (21)-(23).

\subsection{Resonant Scattering}

We return to the case treated in Section 4.1 of the interaction between an incident radiation state and a single molecule, which for simplicity we assume to be linear (like ${ }^{12} \mathrm{CO}$ ). We concentrate on two pairs of photon states of the type $\left|n_{\|}\right\rangle$, $\left|n_{\perp}\right\rangle$ and $\left|n_{\|}^{\prime}\right\rangle,\left|n_{\perp}^{\prime}\right\rangle$ for the incident and scattered radiation, respectively. These states contain $n$ and $n^{\prime}$ photons, while in general $n \neq n^{\prime}$. It is the $n^{\prime}$-photon states $\left|n_{\|}^{\prime}\right\rangle$ and $\left|n_{\perp}^{\prime}\right\rangle$ that are eventually detected by our system to measure polarization.

Since we seek a process that imparts a relative phase shift between such pairs of states, it should be clear that the absorption of a photon followed by a spontaneous emission could not lead to the desired effect. Such a process would randomize any relative 
phase difference between the two linear polarization states at the emission stage. We must therefore move to a higher (second) order mode of interaction. As was mentioned earlier, the best candidate for this is the resonant scattering process where an incident photon is absorbed into a virtual, excited state of the molecule and then re-emitted into a scattered radiation state.

We now denote the initial and final molecule-radiation states as

$$
\begin{gathered}
|i\rangle=|a\rangle \otimes\left|\psi_{j}\right\rangle \\
|f\rangle=\left|a^{\prime}\right\rangle \otimes\left|\psi^{\prime}\right\rangle,
\end{gathered}
$$

with $|a\rangle$ and $\left|a^{\prime}\right\rangle$ the initial and final molecular states, respectively, at sufficiently long times before and after the interaction, while $\left|\psi_{j}\right\rangle$ and $\left|\psi^{\prime}\right\rangle$ are given by

$$
\begin{aligned}
& \left|\psi_{j}\right\rangle=\alpha_{j}\left|n_{\|, j}\right\rangle+\beta_{j}\left|n_{\perp, j}\right\rangle \\
& \left|\psi^{\prime}\right\rangle=\alpha^{\prime} e^{i \phi^{\prime}}\left|n_{\|}^{\prime}\right\rangle+\beta^{\prime}\left|n_{\perp}^{\prime}\right\rangle .
\end{aligned}
$$

In view of our earlier discussion, we have already included the phase factor $e^{i \phi^{\prime}}$ in Equation (28) (see Equation (14)). The scattering amplitude resulting from the interaction with a single molecule can then be determined through (using MKS units; Grynberg et al. 2010; Cohen-Tannoudji et al. 1988)

$$
\begin{aligned}
S_{i f, \ell}= & -i \frac{T}{L^{3}} \frac{\sqrt{n n^{\prime}}}{2 \epsilon_{0} \hbar \sqrt{\omega \omega^{\prime}}} \\
& \times \sum_{b} \frac{\omega_{b a^{\prime}} \omega_{b a}\left\langle a^{\prime}\left|\hat{\mathbf{d}} \cdot \boldsymbol{\epsilon}_{\ell^{\prime}}\right| b\right\rangle\left\langle b\left|\hat{\mathbf{d}} \cdot \boldsymbol{\epsilon}_{\ell}\right| a\right\rangle}{\omega_{b a}-\omega-i \gamma_{b a}},
\end{aligned}
$$

with $\omega\left(\omega^{\prime}\right)$ the frequency of the incident (scattered) radiation, $\omega_{b a}\left(\omega_{b a^{\prime}}\right)$ the resonant frequency between the initial state $|a\rangle$ (final state $\left|a^{\prime}\right\rangle$ ) and virtual state $|b\rangle ; \gamma_{a b}$ is, once again, the relaxation coefficient for a transition between the $|b\rangle$ and $|a\rangle$ states (e.g., for a closed system it equals half the Einstein spontaneous coefficient, $A_{b a} / 2$, when relaxation results only from spontaneous emission). As before, $n$ and $n^{\prime}$ are the number of photons in the initial and final radiation states, respectively, while $\hat{\mathbf{d}}$ is the molecular electric dipole moment operator and $\boldsymbol{\epsilon}_{\ell}$ the unit vector associated to the linear polarization states with $\ell=\|$ or $\perp$. The quantities $T$ and $L^{3}$ are, respectively, the period of interaction between the radiation and the molecule and the fiducial volume of quantization for the radiation field (Grynberg et al. 2010). From now on, we will assume that in Equation (29) the final and initial linear polarization states are the same, as the difference in the energy levels between states of differing polarization (i.e., the Zeeman splitting) will favor similar initial and final polarization states (for a sufficiently long interaction period). This also implies that $\omega_{b a^{\prime}}=\omega_{b a}, \omega=\omega^{\prime}$, and $\left|a^{\prime}\right\rangle=|a\rangle$. Incidentally, we recognize in Equation (29) the same type of second-order interaction term encountered earlier in Equation (24).

It follows that because we expect the relative phase shift to be very small, i.e.,

$$
\phi^{\prime}=\operatorname{Im}\left\{S_{i f, \|}-S_{i f, \perp}\right\} \ll 1,
$$

we should resist the temptation to eliminate seemingly unimportant differences. More precisely, if we write the frequency of the $\sigma$-transitions as

$$
\omega_{ \pm}=\omega_{0} \pm \omega_{Z}
$$

with $\omega_{0}$ and $\omega_{Z}$ the $\pi$-transition frequency and Zeeman splitting, respectively, then we should not approximate $\omega_{ \pm} \simeq \omega_{0}$ on the account that $\omega_{Z} \ll \omega_{0}$. We require $\phi=N_{a} \phi^{\prime} \sim 1$ for the linearto-circular polarization conversion effect to be measurable when the incident radiation state is interacting with a large number $N_{a}$ of molecules. Evidently the size of the volume of interaction is such that the relative phase shift $\phi^{\prime}$ due to the scattering amplitude resulting from the interaction with only one molecule, given by Equation (29), can be extremely small while potentially still sufficient. Taking this into account, we rewrite Equation (29) for the $\pi$ - and $\sigma$-transitions, respectively involving the $\left|n_{\|}\right\rangle$ and $\left|n_{\perp}\right\rangle$ states, with

$$
S_{i f, \|}=-i \sin ^{2}(\iota) \frac{T}{L^{3}} \frac{\sqrt{n n^{\prime}}}{2 \epsilon_{0} \hbar}\left\|\left\langle b_{0}\left|\hat{d}_{\|}\right| a\right\rangle\right\|^{2} \frac{\omega_{0}^{2}}{\omega\left(\omega_{0}-\omega-i \gamma_{b_{0} a}\right)}
$$

$$
\begin{aligned}
S_{i f, \perp}= & -i \sin ^{2}(\iota) \frac{T}{L^{3}} \frac{\sqrt{n n^{\prime}}}{2 \epsilon_{0} \hbar}\left\|\left\langle b_{ \pm}\left|\hat{d}_{\perp}\right| a\right\rangle\right\|^{2} \\
& \times \frac{\left(\omega_{0} \pm \omega_{Z}\right)^{2}}{\omega\left(\omega_{0} \pm \omega_{Z}-\omega-i \gamma_{b_{ \pm} a}\right)},
\end{aligned}
$$

where the summation on the virtual states was removed on the account that one state $\left|b_{i}\right\rangle$ (with $i=0, \pm$ ) will dominate independently for each transition because of the strong resonance (note that $\omega \gg \omega_{Z} \gg \gamma_{b_{i} a}$ for the transitions considered here). We have used this notation for the virtual states $\left|b_{i}\right\rangle$ in Equations (32) and (33) to underline the different types of transitions (i.e., $\pi$-transitions bring no change in magnetic quantum number and obey $\Delta m_{J}=0$, while $\sigma$-transitions verify $\Delta m_{J}= \pm 1$, hence the notation). Also, the inclination angle of the magnetic field relative to the scattering propagation direction (or the line of sight to the observer) is given by $\iota$, from which $\hat{\mathbf{d}} \cdot \boldsymbol{\epsilon}_{\ell}=\hat{d}_{\ell} \sin (\iota)$ for the two states of linear polarization. In the numerical calculations presented in the next section we will set $\iota=\pi / 2$, effectively setting the magnetic field in the plane of the sky.

If we now account for the population of molecules with which the radiation interacts, then we must also consider the fact that their spectrum (or velocity) will be spread over some normalized distribution function $h(\omega)$. We can also substitute $T=l / c$ in the same equations, where $l$ is the size of the region of interaction. We then have at the frequency $\omega$ of the incident and scattered photons

$$
\begin{aligned}
\phi(\omega) \simeq & -\sin ^{2}(\iota) \frac{l N_{a} \sqrt{u(\omega) u^{\prime}(\omega)}}{2 \epsilon_{0} c \hbar^{2} \omega^{2}} \\
& \times\left\{\left\|\hat{d}_{\|, b a}\right\|^{2} \int \frac{x^{2}(x-\omega)}{(x-\omega)^{2}+\gamma_{b_{0} a}^{2}} h(x) d x\right. \\
- & \left\|\hat{d}_{\perp, b a}\right\|^{2} \int\left[\frac{\left(x+\omega_{Z}\right)^{2}\left(x+\omega_{Z}-\omega\right)}{\left(x+\omega_{Z}-\omega\right)^{2}+\gamma_{b_{+} a}^{2}}\right. \\
& \left.\left.+\frac{\left(x-\omega_{Z}\right)^{2}\left(x-\omega_{Z}-\omega\right)}{\left(x-\omega_{Z}-\omega\right)^{2}+\gamma_{b_{-} a}^{2}}\right] h(x) d x\right\},
\end{aligned}
$$

where $N_{a}$ is the number of molecules in state $|a\rangle$ and $u(\omega)$ is the radiation energy density at frequency $\omega$. For numerical calculations the terms $\left\|\hat{d}_{\ell, b a}\right\|^{2}=\left\|\left\langle b_{i}\left|\hat{d}_{\ell}\right| a\right\rangle\right\|^{2}(i=0, \pm$ and $\ell=\|, \perp)$ 
can advantageously be related to the corresponding Einstein spontaneous emission coefficient with (Cohen-Tannoudji et al. 1988)

$$
A_{\ell, b a}=\frac{\omega_{b a}^{3}\left\|\hat{d}_{\ell, b a}\right\|^{2}}{3 \pi \epsilon_{0} \hbar c^{3}} .
$$

Notably, for ${ }^{12} \mathrm{CO}$ we have $\left\|\hat{d}_{\|, b a}\right\|=\sqrt{2}\left\|\hat{d}_{\perp, b a}\right\|$ and $A_{b_{0} a} \simeq$ $2 A_{b_{ \pm} a}$. It is therefore apparent from Equation (34) that any relative phase shift would have vanished had we approximated $\omega_{0} \pm \omega_{Z} \simeq \omega_{0}$.

\subsubsection{Circular Polarization of the ${ }^{12} \mathrm{CO}(\mathrm{J}=2 \rightarrow 1)$ Transition in Orion $K L$}

Let us now provide an estimate of the importance of the effect for the ${ }^{12} \mathrm{CO}(J=2 \rightarrow 1)$ transition in Orion KL. The region of interaction $l$ will be constrained by the lifetime of the transition, which is determined by the relaxation rate $\gamma_{b a}$, or the effective mean free path of a photon. Plume et al. (2012) determined from observations of $\mathrm{CO}$ isotopologues that for Orion KL (in the Hot Core) the hydrogen number density is $n_{\mathrm{H}_{2}}=10^{7} \mathrm{~cm}^{-3}$ and the temperature $T_{\mathrm{ex}}=150 \mathrm{~K}$, which together yield a collisional quenching rate for ${ }^{12} \mathrm{CO}$ of

$$
\gamma_{21} \simeq n_{\mathrm{H}_{2}}\langle\sigma v\rangle \approx 1.3 \times 10^{-3} \mathrm{~s}^{-1} .
$$

This is more than three orders of magnitude greater than $A_{21}^{\mathrm{CO}}=$ $7 \times 10^{-7} \mathrm{~s}^{-1}$, and we will adopt this value for the corresponding relaxation rate, i.e., $\gamma_{b a} \equiv \gamma_{21}$ (at the specified temperature the momentum-rate transfer coefficient $\langle\sigma v\rangle \approx 10^{-10} \mathrm{~cm}^{3} \mathrm{~s}^{-1}$; see Shull \& Draine 1990). Accordingly, the associated region of interaction equals $l_{\gamma} \approx c / \gamma_{21} \approx 2.3 \times 10^{13} \mathrm{~cm}$. The effective mean path of a photon will depend on the absorption coefficient $\alpha_{\omega}$ and the resonant scattering coefficient $\sigma_{\omega}$ at the frequency $\omega$ of the photon. The absorption coefficient (Rybicki \& Lightman 1979) is given by

$$
\alpha_{\omega}=\frac{n_{\mathrm{CO}} g_{2} e^{-E_{1} / k T_{\mathrm{ex}}}}{Q_{\mathrm{CO}}\left(T_{\mathrm{ex}}\right)} \frac{\pi^{2} c^{2} A_{21}^{\mathrm{CO}}}{\omega^{2}} \frac{\hbar \omega}{k T_{\mathrm{ex}}} h(\omega)
$$

after integrating over the molecular population of the lower state, with $g_{2}=5$ the degeneracy of the upper state, $E_{1}$ the energy of the lower state $\left(E_{1} / k \simeq 5.5 \mathrm{~K}\right.$, with $k$ the Boltzmann constant), $n_{\mathrm{CO}} \approx 10^{3} \mathrm{~cm}^{-3}$ the density of ${ }^{12} \mathrm{CO}$ (i.e., a relative abundance of approximately $\left.10^{-4}\right)$, and $Q_{\mathrm{CO}}\left(T_{\mathrm{ex}}\right)=54.6$ its partition function at $T_{\mathrm{ex}}=150 \mathrm{~K}$ (Pickett et al. 1998).

The resonant scattering coefficient is obtained through a similar integration of the resonant scattering cross section (Grynberg et al. 2010) over the molecular population of the lower state, which yields

$$
\sigma_{\omega}=\frac{n_{\mathrm{CO}} g_{1} e^{-E_{1} / k T_{\mathrm{ex}}}}{Q_{\mathrm{CO}}\left(T_{\mathrm{ex}}\right)} \frac{3 c^{2}}{\omega^{2}} 4 \pi^{3} \gamma_{21} h(\omega),
$$

with the degeneracy of the lower state $g_{1}=3$. If we use a Gaussian distribution for the spectral profile at its maximum, i.e., $h(\omega)=1 /(\sqrt{2 \pi} \Delta \omega)$, with $\Delta \omega=9.4 \times 10^{7} \mathrm{rad} \mathrm{s}^{-1}$ (i.e., a standard deviation of approximately $20 \mathrm{~km} \mathrm{~s}^{-1}$; see below) and $\omega=1.4 \times 10^{12} \mathrm{rads}^{-1}$ (i.e., $230.5 \mathrm{GHz}$ ) we find that $\alpha_{\omega}=8.1 \times 10^{-17} \mathrm{~cm}^{-1}$ and $\sigma_{\omega}=4.7 \times 10^{-11} \mathrm{~cm}^{-1}$. The effective mean path is then determined through (Rybicki \& Lightman 1979)

$$
\begin{aligned}
l_{\mathrm{mp}} & =\left[\alpha_{\omega}\left(\alpha_{\omega}+\sigma_{\omega}\right)\right]^{-1 / 2} \\
& \simeq 1.6 \times 10^{13} \mathrm{~cm} .
\end{aligned}
$$

We therefore find that both path lengths $l_{\gamma}$ and $l_{\mathrm{mp}}$ are of similar sizes and little change would occur whether we use one or the other. However, it is the case that $l_{\mathrm{mp}} \lesssim l_{\gamma}$, and we therefore set $l=l_{\mathrm{mp}}$ for the size of the region of interaction in Equation (34), which we rewrite here for ${ }^{12} \mathrm{CO}(J=2 \rightarrow 1)$ :

$$
\begin{aligned}
\phi_{21}^{\mathrm{CO}}(\omega) \simeq & -\sin ^{2}(\iota) l_{\mathrm{mp}}^{4} \frac{n_{\mathrm{CO}} g_{1} e^{-E_{1} / k T_{\mathrm{ex}}}}{Q_{\mathrm{CO}}\left(T_{\mathrm{ex}}\right)} \frac{3 \pi c^{2} A_{21}^{\mathrm{CO}}}{4 \hbar \omega_{0}^{3} \omega^{2}} \\
& \times \sqrt{u(\omega) u^{\prime}(\omega)} \iint \frac{x^{2}(x-\omega)}{(x-\omega)^{2}+\gamma_{21}^{2}} h(x) d x \\
& -\frac{1}{2} \int\left[\frac{\left(x+\omega_{Z}\right)^{2}\left(x+\omega_{Z}-\omega\right)}{\left(x+\omega_{Z}-\omega\right)^{2}+\gamma_{21}^{2}}\right. \\
& \left.\left.+\frac{\left(x-\omega_{Z}\right)^{2}\left(x-\omega_{Z}-\omega\right)}{\left(x-\omega_{Z}-\omega\right)^{2}+\gamma_{21}^{2}}\right] h(x) d x\right\} .
\end{aligned}
$$

In this equation the volume of the region of interaction was set to $\approx l_{\mathrm{mp}}^{3}$.

It is interesting to note that the integrals in Equation (40) can be combined and transformed such that

$$
\begin{aligned}
\phi_{21}^{\mathrm{CO}}(\omega) \simeq & \omega_{Z}^{2} \sin ^{2}(\iota) l_{\mathrm{mp}}^{4} \frac{n_{\mathrm{CO}} g_{1} e^{-E_{1} / k T_{\mathrm{ex}}}}{Q_{\mathrm{CO}}\left(T_{\mathrm{ex}}\right)} \\
& \times \frac{3 \pi c^{2} A_{21}^{\mathrm{CO}}}{4 \hbar \omega_{0}^{3} \omega^{2}} \sqrt{u(\omega) u^{\prime}(\omega)} I(\omega),
\end{aligned}
$$

where

$$
\begin{aligned}
I(\omega)= & \int\left\{\frac{x^{2}(x-\omega)\left[3(x-\omega)^{2}-\gamma_{21}^{2}-\omega_{Z}^{2}\right]}{\left[(x-\omega)^{2}+\gamma_{21}^{2}\right]}\right. \\
& \left.+(x-\omega)\left(\omega^{2}-3 x^{2}\right)+\gamma_{21}^{2}(3 x-\omega)+\omega_{Z}^{2}(x+\omega)\right\} \frac{h(x)}{\Delta} d x,
\end{aligned}
$$

with $\Delta=\left[\left(x+\omega_{Z}-\omega\right)^{2}+\gamma_{21}^{2}\right]\left[\left(x-\omega_{Z}-\omega\right)^{2}+\gamma_{21}^{2}\right]$. We thus find that the effect, or the relative phase shift $\phi_{21}^{\mathrm{CO}}$, is proportional to the square of the magnitude of the plane of the sky component of the magnetic field from the presence of the term $\omega_{Z}^{2} \sin ^{2}(\iota)$ in Equation (41).

For the 10.4 m CSO telescope with an efficiency of approximately $60 \%$, we can convert the antenna temperature to energy density according to

$$
u(\omega)=5.42 \times 10^{-22} \frac{T_{\mathrm{A}}^{*} \gamma_{21}}{c} \approx 2.3 \times 10^{-35} T_{\mathrm{A}}^{*} \mathrm{erg} \mathrm{cm}^{-3}
$$

while our circular and linear polarization spectra shown in Figures 1 and 3 yield $u(\omega) \approx u^{\prime}(\omega)$ and $T_{\mathrm{A}}^{*} \lesssim 0.2 \mathrm{~K}$ in the line wings where polarization is detected.

Using the parameter values already listed before, as well as $\iota=\pi / 2$ and $\omega_{Z} \approx 1.3 \mathrm{rad} \mathrm{s}^{-1}$ (i.e., $\approx 0.2 \mathrm{~Hz}$, with $B \approx 1 \mathrm{mG}$ (Crutcher et al. 1999; Houde et al. 2009) and $g_{J}^{\mathrm{CO}} \simeq$ -0.269 (Gordy \& Cook 1984)), we numerically integrated Equations (41) and (42) over a Gaussian profile of the form

$$
h(x)=\frac{1}{\sqrt{2 \pi} \Delta \omega} e^{-\frac{1}{2}\left(\frac{x-\omega_{0}}{\Delta \omega}\right)^{2}}
$$

chosen to approximately match the width of our observed ${ }^{12} \mathrm{CO}(J=2 \rightarrow 1)$ spectra shown in Figures 1 and 3 . We also used a linear radiation energy density profile $u(\omega)$ similar to 


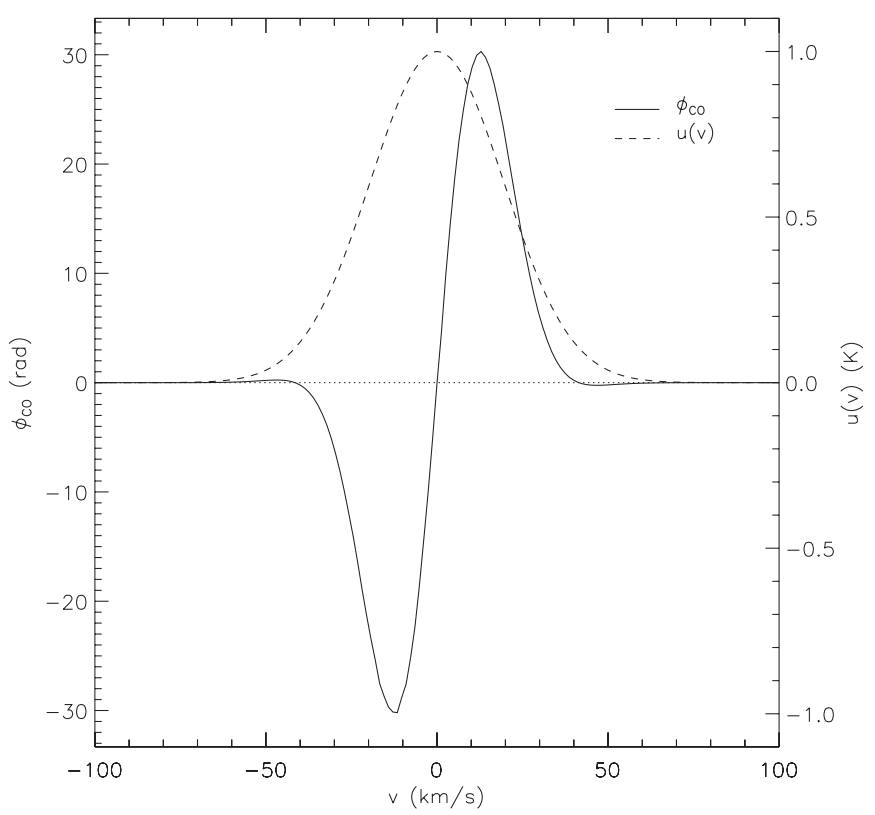

Figure 4. Numerical calculations of the expected relative phase shift $\phi_{21}^{\mathrm{CO}}$ (solid curve) for ${ }^{12} \mathrm{CO}(J=2 \rightarrow 1)$ in Orion $\mathrm{KL}$ based on our resonant scattering model, using the left vertical scale. The magnetic field strength was set to $1 \mathrm{mG}$. The underlying linear polarization radiation profile $u(v)$ is shown with the dashed curve, using the vertical scale on the right.

$h$ but with a peak temperature $T_{\mathrm{A}}^{*}=1 \mathrm{~K}$. The result is shown in Figure 4, where we find that, for this set of parameters, the relative phase shift induced by the resonant scattering process is potentially significant for ${ }^{12} \mathrm{CO}(J=2 \rightarrow 1)$ with a maximum value for $\phi_{21}^{\mathrm{CO}}$ of approximately $30 \mathrm{rad}$. It is, however, important to realize that these calculations are uncertain by a large amount in view of the strong dependency of $\phi_{21}^{\mathrm{CO}}$ on some parameters. For example, a close look at Equation (41) reveals that $\phi_{21}^{\mathrm{CO}}$ varies inversely with the fourth power of the gas density $n_{\mathrm{H}_{2}}$ and the third power of the ${ }^{12} \mathrm{CO}$ abundance; an increase of only a factor of two for these parameters would bring the maximum

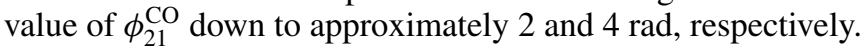

The behavior of $\phi_{21}^{\mathrm{CO}}$ is different if the gas density decreases to the point where the region of interaction is defined by $l_{\gamma}$ instead of $l_{\mathrm{mp}}$ (see Equations (36)-(39) and the related discussion). We then find that the relative phase shift varies inversely with the second power of $n_{\mathrm{H}_{2}}$ and is proportional to the ${ }^{12} \mathrm{CO}$ abundance (the same dependencies apply to other molecules with lower abundances than ${ }^{12} \mathrm{CO}$ ). Likewise, $\phi_{21}^{\mathrm{CO}}$ is also strongly dependent on the excitation temperature (mainly through the partition function, with the inverse dependency of the molecular abundance) and the magnetic field strength (proportional to its second power through the Zeeman splitting). Whatever the case, it is apparent that the linear-to-circular polarization conversion effect is strongly affected by even modest changes on a range of parameters, and within their established uncertainties. For the present case, although there are several combinations that would allow us to match the strength of the polarization conversion effect found in our calculations to the level of circular polarization we observe for ${ }^{12} \mathrm{CO}(J=2 \rightarrow 1)$ in Orion $\mathrm{KL}$, we will simply reduce the magnetic field strength from $1 \mathrm{mG}$ to $0.1 \mathrm{mG}$ (resulting in a Zeeman splitting $\omega_{Z} \simeq 0.02 \mathrm{~Hz}$ ) and leave all other parameters unchanged. Although this value for the magnetic field strength is reasonable in general for star-forming regions at such gas densities, there is no guarantee that it is right for this source.

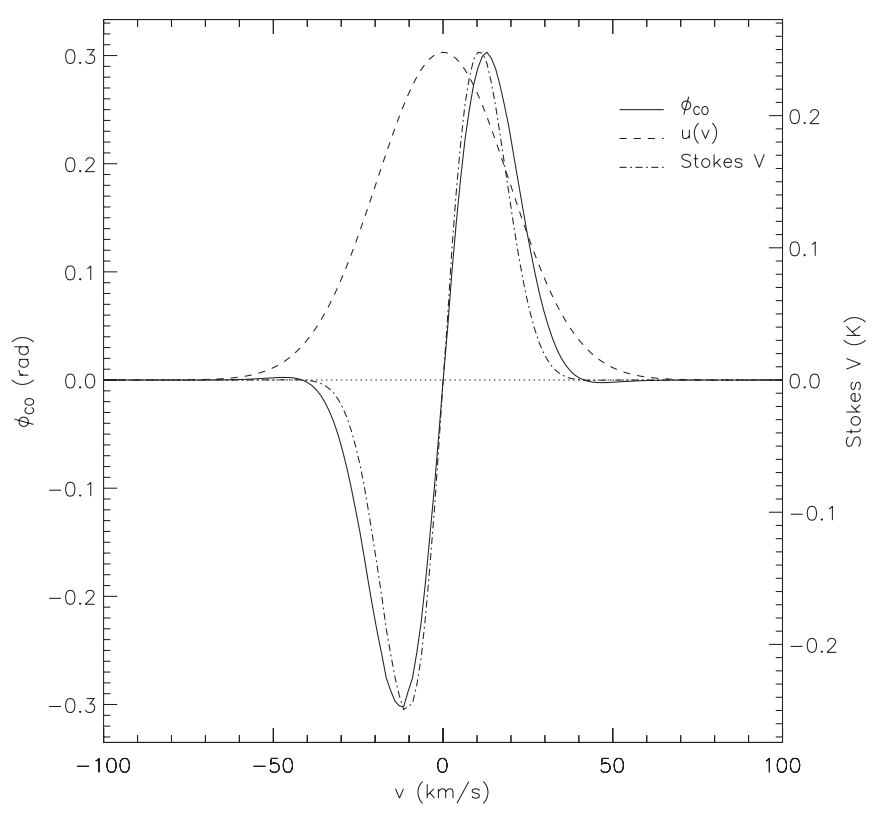

Figure 5. Numerical calculations of the expected relative phase shift $\phi_{21}^{\mathrm{CO}}$ (solid curve) for ${ }^{12} \mathrm{CO}(J=2 \rightarrow 1)$ in Orion KL based on our resonant scattering model, using the left vertical scale. The underlying linear polarization radiation profile $u(v)$ is shown with the dashed curve, has been normalized in the figure, but has a peak antenna temperature of $1 \mathrm{~K}$. The corresponding Stokes $V$ spectrum is also shown (dot-dashed curve), using the vertical scale on the right. This spectrum is proportional to $u(v) \sin \left(\phi_{21}^{\mathrm{CO}}\right)$. For this simulation and the other that follows, the magnetic field strength was set to $0.1 \mathrm{mG}$.

The corresponding results can be found in Figure 5, where the expected relative phase shift $\phi_{21}^{\mathrm{CO}}$ for ${ }^{12} \mathrm{CO}(J=2 \rightarrow 1)$ based on our resonant scattering model (solid curve; left vertical scale), the underlying linear polarization radiation profile $u(v)$ (dashed curve), and the corresponding Stokes $V$ spectrum (dot-dashed curve; right vertical scale) are shown. The Stokes $V$ spectrum is proportional to $u(v) \sin \left(\phi_{21}^{\mathrm{CO}}\right.$ ), and we set $2 \alpha_{j} \beta_{j}=1$ (see Equation (5)). Its maximum amplitude is now in line with that of our observations shown in Figure 1. We will discuss Figure 5 in more detail, especially its shape, in Section 5.

The previous discussion raises the question as to what we should expect observations to reveal in cases where $\phi_{21}^{\mathrm{CO}}$ becomes very high. For example, measurements conducted with ${ }^{12} \mathrm{CO}(J=2 \rightarrow 1)$ in molecular clouds will commonly probe gases of significantly lower densities than is the case for Orion KL. Although changes in other parameters (e.g., the excitation temperature and the magnetic field strength) could somewhat offset the effect of a lower gas density, it is likely that under such conditions the relative phase shift $\phi_{21}^{\mathrm{CO}}$ will reach significantly higher values (perhaps as much as several hundred radians). The fact that the Stokes $V$ spectrum is proportional to $\sin \left(\phi_{21}^{\mathrm{CO}}\right)$ will bring strong oscillations as a function of the frequency (or velocity), which will then result in a cancellation of any circular polarization across the spectrum (given a finite spectral resolution). This cancellation will likely be accentuated if several incident linear polarization modes are resonantly scattered into the telescope beam. Finally, as a result of the Stokes $u$ dependency on $\cos \left(\phi_{21}^{\mathrm{CO}}\right)$ (see Equation (8)), only linear polarization parallel or perpendicular to the magnetic field will be detected (i.e., Stokes $q$ in Equation (7)).

\subsubsection{Circular Polarization of $H C N$ and $H N C O$ in Orion $K L$}

Evidently, our model applies equally to other linear molecules and should therefore account for the lack of circular polarization 
in our $\mathrm{HCN}(J=3 \rightarrow 2)$ spectrum of Figure 2 . For that molecule, its lower abundance, i.e., $\approx 10^{-7}$, implies that ${ }^{5} l=$ $l_{\gamma} \approx 1.7 \times 10^{13} \mathrm{~cm}$ in Equation (34) under similar conditions as for ${ }^{12} \mathrm{CO}$ (Blake et al. 1987; Schilke et al. 2001), leaving the corresponding contribution to $\phi(\omega)$ basically unchanged. The reduction in molecular abundance is practically canceled out by an increase of approximately the same factor in the Einstein spontaneous emission (i.e., $A_{32}^{\mathrm{HCN}} \simeq 8 \times 10^{-4} \mathrm{~s}^{-1}$ ) in the numerator of the same equation. However, the Zeeman sensitivity of $\mathrm{HCN}$ is approximately three times less than that of ${ }^{12} \mathrm{CO}$ (i.e., $g_{J}^{\mathrm{HCN}} \simeq-0.0962$, from Gordy \& Cook 1984), which implies a corresponding loss of about an order of magnitude for $\phi(\omega)$. Taking all these factors into account, as well as a decrease of about a factor of four for $u(\omega)$, and the increases in $\omega_{0}$ and the partition function $\left(Q_{\mathrm{HCN}}(150 \mathrm{~K}) \simeq 210\right)$, we find that the effect for $\mathrm{HCN}$ ( $J=3 \rightarrow 2$ ) should be approximately two orders of magnitude weaker than for ${ }^{12} \mathrm{CO}(J=2 \rightarrow 1)$, again assuming similar physical conditions for the two molecular species. This is consistent with our results of Figure 2. Moreover, it is likely that the gas density probed by this molecular species is higher given its significant critical density $\left(\approx 10^{7} \mathrm{~cm}^{-3}\right)$. An increase in $n_{\mathrm{H}_{2}}$ by only a factor of a few would further reduce any circular polarization to even lower levels.

The case of the $\mathrm{HNCO} / \mathrm{HN}^{13} \mathrm{CO}\left(N_{K_{a} K_{c}}=12_{1,12} \rightarrow 11_{1,11}\right)$ transitions is more difficult to analyze. These molecules are asymmetric tops and will possess more complicated Zeeman spectra than linear molecules like $\mathrm{CO}$ and $\mathrm{HCN}$ (we concentrate on HNCO in what follows). We know, however, that the abundance of $\mathrm{HNCO}$ (i.e., $10^{-8}$ to $10^{-9}$ ) is less than CO by a factor of 4-5 orders of magnitude (Tideswell et al. 2010) and its Einstein spontaneous coefficient is comparable to $\mathrm{HCN}(J=3 \rightarrow 2)$ with $A^{\mathrm{HNCO}}=3 \times 10^{-4} \mathrm{~s}^{-1}$ for these transitions. However, the Zeeman sensitivity is likely to be significantly higher as its electronic ground state possesses electronic spin. More precisely, the upper and lower energy levels involved in the transitions discussed here are part of triplet states with electronic spin $S=1$ (Pickett et al. 1998). The associated electronic spin contribution to the Landé factor can be approximated with (Gordy \& Cook 1984)

$$
g_{J}^{\mathrm{HNCO}} \simeq \frac{J(J+1)+S(S+1)-N(N+1)}{J(J+1)},
$$

which for the strongest of these lines, i.e., when $\Delta J=+1$ (Pickett et al. 1998), covers a range of -0.182 to 0.167 . The Zeeman splitting between corresponding $\pi$ - and $\sigma$-lines can be approximated by multiplying these Landé factors by the Bohr magneton. Taking into account the fact that the nuclear magneton is used instead of the Bohr magneton for similar calculations with ${ }^{12} \mathrm{CO}$ (and $\mathrm{HCN}$ ), we expect the Zeeman splitting of the strongest $\operatorname{HNCO}\left(N_{K_{a} K_{c}}=12_{1,12} \rightarrow\right.$ $11_{1,11}$ ) lines to be roughly a thousand times larger than for ${ }^{12} \mathrm{CO}$. This would lead to an increase of approximately six orders of magnitude in the value of $\omega_{Z}^{2}$ in Equation (34). Combining these changes (i.e., in molecular abundance, Einstein spontaneous coefficient, and Zeeman sensitivity) with those for the partition function $\left(Q_{\mathrm{HNCO}}(150 \mathrm{~K}) \simeq 2800\right.$, about 50 times that of $\mathrm{CO}$ ) and the radiation energy density (a reduction

\footnotetext{
5 Because of the larger value of the Einstein spontaneous emission coefficient for this transition, we set $\gamma_{\mathrm{HCN}} \simeq \gamma_{21}+A_{32}^{\mathrm{HCN}} / 2$ for the relaxation rate (Grynberg et al. 2010); the value thus obtained for $l_{\gamma}$ is slightly smaller than that for ${ }^{12} \mathrm{CO}(J=2 \rightarrow 1)$, where the Einstein spontaneous emission coefficient could safely be neglected.
}

of approximately 40 for similar levels of linear polarization), we would expect the linear-to-circular polarization conversion effect to be approximately as strong for $\mathrm{HNCO}\left(N_{K_{a} K_{c}}=\right.$ $\left.12_{1,12} \rightarrow 11_{1,11}\right)$ as for ${ }^{12} \mathrm{CO}(J=2 \rightarrow 1)$. Although there is a significant level of uncertainty in the previous calculations, this result is also consistent with our observations (Figure 2).

\section{DISCUSSION}

The analysis presented in the previous section established that the relative phase shift induced by the resonant scattering process between linear polarization components parallel and perpendicular to the plane of the sky component of the magnetic field can account for the levels of circular polarization detected in the spectra presented in Figures 1 and 2. It may be surprising that a Zeeman splitting on the order of $0.1 \mathrm{~Hz}$ or less could be responsible for such a significant effect across a spectral line that is on the order of $10 \mathrm{MHz}$ wide and centered at $230.5 \mathrm{GHz}$. But in the case of ${ }^{12} \mathrm{CO}(J=2 \rightarrow 1)$ the conversion of linear to circular polarization is likely to be efficient in Orion KL, although we again emphasize that small changes in some of the main parameters (e.g., $\omega_{Z}, l_{\mathrm{mp}}$, or $n_{\mathrm{CO}}$ ) can significantly affect our results. There is one aspect, however, that requires further discussion: the expected profile of the circular polarization Stokes $V$ spectrum.

As was mentioned earlier, Figure 5 also shows the Stokes $V$ profile that results from the numerical calculations of $\phi_{21}^{\mathrm{CO}}$ discussed in Section 4.3.1 (the dot-dashed curve, using the vertical scale in the right side of the graph). We approximated this spectrum with the following function (in units of Kelvin):

$$
V(\omega)=e^{-\frac{1}{2}\left(\frac{\omega-\omega_{0}}{\Delta \omega}\right)^{2}} \sin \left[\phi_{21}^{\mathrm{CO}}(\omega)\right],
$$

i.e., we set $2 \alpha_{j} \beta_{j} \approx 1$ (see Equations (5) and (27)) and, as is apparent in Equation (46), used a Gaussian profile for $u(\omega)$ with a peak antenna temperature of $1 \mathrm{~K}$ for the radiation associated with the $\left|n_{\|}\right\rangle$and $\left|n_{\perp}\right\rangle$ incident states. We also converted the abscissa to a velocity scale to ease the comparison with the observed spectrum of Figure 1. The most obvious discrepancy between the two spectra is the fact that our calculations yield an antisymmetric profile, while the observations do not. In fact, the calculated profile is not unlike the typical Stokes $V$ spectrum expected from Zeeman sensitive molecular species and transitions. A detailed comparison between calculations and observations is complicated by the fact that our model contains several approximations. For example, the Gaussian profile chosen for our calculations does not perfectly match that of the measured linear polarization spectrum of Figure 3, and the cores of the observed polarization line profiles (linear and circular) are very likely dominated by instrumental polarization, which our model does not consider. But it is important to realize that several factors can affect the shape of the calculated Stokes $V$ spectrum:

1. The perfect antisymmetry seen in the results of Figure 5 stems from the symmetry of the underlying Gaussian used for the calculations. This is made clearer in Figure 6, where another calculation for the same parameters used for Figure 5 is shown, but with the Gaussian profile of Equations (44) and (46) replaced with a slightly uneven line shape. The result is a circular polarization spectrum that is markedly broader on one side (where $v \lesssim 15 \mathrm{~km} \mathrm{~s}^{-1}$ ) than the other. The same would happen for a typical Stokes $V$ spectrum from a Zeeman sensitive transition for a slightly 
uneven Stokes $I$ profile, since they are linked through a derivative. But it is important to note that, in our case, it is the line profile of the incident (background) linear polarization radiation that is in question, which may be different from the Stokes I spectrum.

2. In the calculations of the integral contained in Equation (34) (or (42)) we assumed that a photon at a frequency $\omega$ will scatter off all molecules at any other frequencies covered under the profile $h(\omega)$. We must realize that this is not likely to be the case since the size of the molecule-radiation region of interaction is relatively small (i.e., $\sim 10^{13} \mathrm{~cm}$ or $\sim 10^{-5}$ pc; see Equation (39)) whereas molecules belonging to different velocity ranges are likely to be separated by larger distances within Orion $\mathrm{KL}$ (the ${ }^{12} \mathrm{CO}(J=2 \rightarrow 1)$ spectrum covers a velocity range of $\left.\approx \pm 50 \mathrm{~km} \mathrm{~s}^{-1}\right)$. This could significantly affect the shape of the resulting Stokes $V$ spectrum.

3. The numerical calculations that produced Figure 5 considered a single incident radiation mode. But we know from the related discussion in Section 4.1 (see Equations (13)-(20)) that even at a single frequency several incident modes, coming from different orientations with potentially differing linear polarization states, take part in the resonant scattering process. This brings an averaging process that will certainly affect the line shape of the circular polarization profile.

4. There also exists an averaging process due to the size of the telescope beam. At the distance of Orion KL (i.e., approximately $450 \mathrm{pc}$ ) our telescope beam of $\approx 32^{\prime \prime}$ covers a region of approximately $0.07 \mathrm{pc}$ or $10^{17} \mathrm{~cm}$, which is several orders of magnitude larger than the region of interaction. Our observations therefore contain contributions from several radiation-molecule interaction regions, where key parameters that affect the Stokes $V$ line profile are likely to vary.

Although these considerations reveal the complexity of the problem and that we perhaps should not expect our observations to closely match the profile resulting from our simplified model and presented in Figure 5, it is nevertheless possible to find relatively simple conditions that would allow us to calculate Stokes $V$ profiles that are consistent with our circular polarization detection in ${ }^{12} \mathrm{CO}(J=2 \rightarrow 1)$.

To do so, we consider incident radiation modes for which $\theta(v)$ varies with velocity (frequency) and possesses some distribution about the orientation of the foreground magnetic field on the plane of the sky. Since the linear-to-circular conversion of polarization will only occur when $\theta(v) \neq 0$ (and $\pi / 2$ ), it is possible that most linear polarization signals at frequencies satisfying this condition will be efficiently converted to circular polarization, leaving only a well-defined orientation angle (i.e., $\theta=0$ ) for the outgoing linear polarization radiation. Our linear polarization spectrum of Figure 3 indeed shows a well-defined polarization angle across the spectral line. If the distribution of $\theta(v)$ is not uniform about $\theta=0$ and, for example, is positive in some regions and negative in others, then the shape of the circular polarization spectrum would clearly depart from the asymmetric profile displayed in Figure 5 (or even 6). This is exemplified in Figure 7, where have computed the Stokes $V$ spectrum for conditions similar to those used for Figure 5, with the exception that the angle $\theta(v)$ between the incident linear polarization and the magnetic field orientation varies linearly with velocity at a rate of $d \theta / d v=-1 \mathrm{deg} /\left(\mathrm{km} \mathrm{s}^{-1}\right)$ (with $\theta=0$ at $v=0)$. The resulting Stokes $V$ spectrum follows from Equations (5) and (41) (and Equation (46)). It is then found that

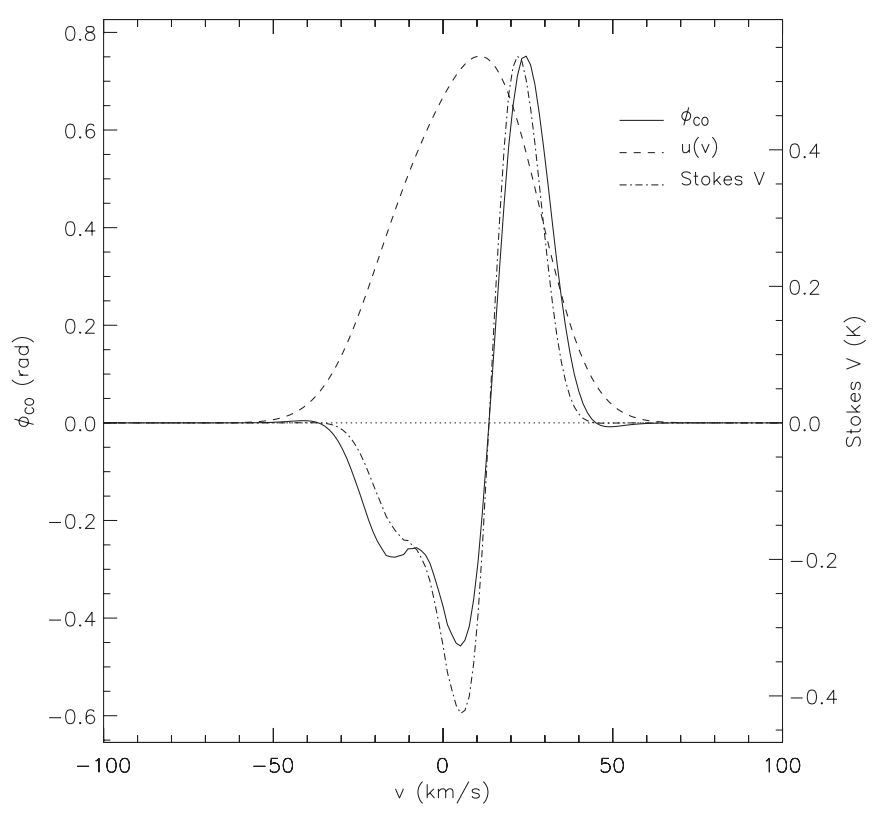

Figure 6. Numerical calculations of the expected relative phase shift $\phi_{21}^{\mathrm{CO}}$ (solid curve) for ${ }^{12} \mathrm{CO}(J=2 \rightarrow 1)$ in Orion $\mathrm{KL}$ (using the left vertical scale) for a slightly uneven underlying (normalized) linear polarization profile $u(v)$ of $1 \mathrm{~K}$ peak antenna temperature (dashed curve). The corresponding Stokes $V$ spectrum is also shown (dot-dashed curve), using the vertical scale on the right. Note the change in the resulting line profile when compared with Figure 5. The magnetic field strength was set to $0.1 \mathrm{mG}$.

the presence of the factor $\alpha_{j}=\sin (\theta)$ in this equation results in a symmetric profile about $v=0$ that is consistent with our circular polarization measurements on ${ }^{12} \mathrm{CO}(J=2 \rightarrow 1)$. As stated above, the core of this spectral line is very likely dominated by instrumental polarization, and the true polarization level there should be zero or close to zero, as in our Figure 7. Although this does not ensure that our model can perfectly account for our observations across the whole spectrum, the results of Figure 7 are consistent with our measurements away from the core of the spectral line where signals in the Stokes $V$ profile have the same sign.

We also note that the levels of circular polarization predicted by our model are likely to be consistent with the IK Tau SiO $v=1, v=2,(J=1 \rightarrow 0)$ (at $43.1 \mathrm{GHz}$ and $42.8 \mathrm{GHz}$, respectively) observations of Cotton et al. (2011). Although a more precise analysis would be necessary to ensure this (we are not, for example, accounting for possible maser saturation), we can make an approximate assessment of the strength of the effect for $\mathrm{SiO}$ relative to $\mathrm{CO}$ using our previous calculations. If we take the $\mathrm{SiO} v=2,(J=1 \rightarrow 0), 42.8 \mathrm{GHz}$ transition as an example, we know that although the abundance is one to two orders of magnitude less than ${ }^{12} \mathrm{CO}$ (Decin et al. 2012), its Einstein spontaneous coefficient is about an order of magnitude greater at $A \simeq 4 \times 10^{-6} \mathrm{~s}^{-1}$, while its Zeeman sensitivity is only a factor of two or so lower (Davis \& Muenter (1974) find $g_{J}^{\mathrm{SiO}} \simeq-0.154$ to -0.155 for $v=0,1$, and 2). If we add to this the fact that the frequency of $\mathrm{SiO} v=2,(J=1 \rightarrow 0)$ is less than five times that of ${ }^{12} \mathrm{CO}(J=2 \rightarrow 1)$ and set the spectral line width to match their observations (on the order of $1 \mathrm{~km} \mathrm{~s}^{-1}$ ), then we find that the polarization conversion effect is likely to be important for $\mathrm{SiO}$ for a significant range of gas densities and magnetic field strengths. A similar result is expected for the $\mathrm{SiO} v=1$, $(J=1 \rightarrow 0), 43.1 \mathrm{GHz}$ transition. These calculations could then resolve the known problem of high circular polarization 


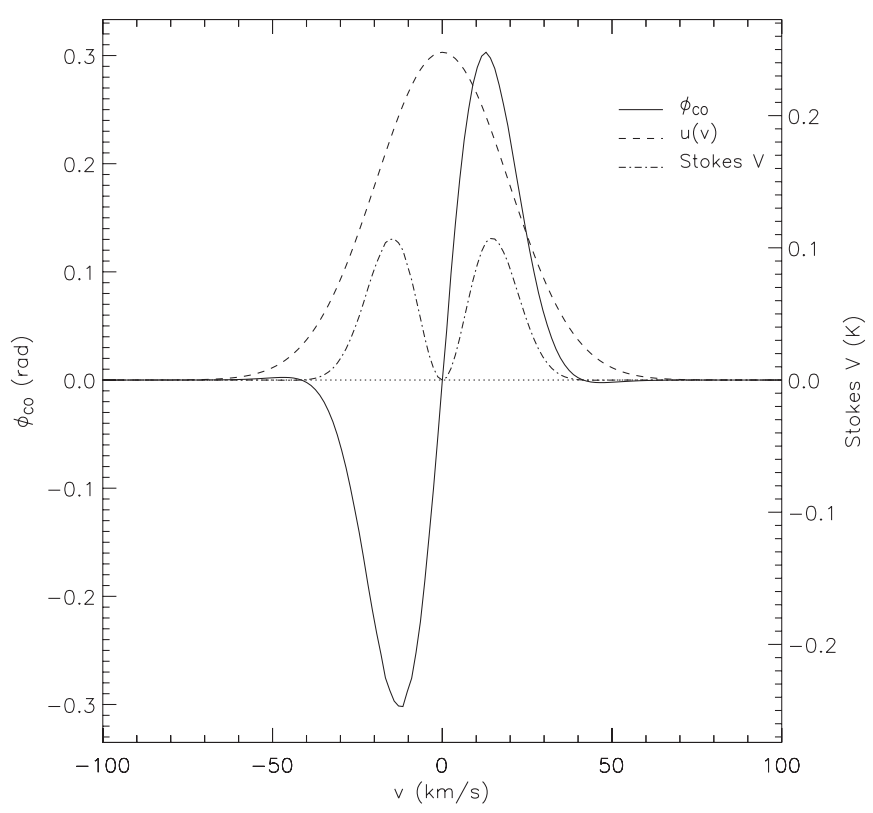

Figure 7. Numerical calculations of the expected relative phase shift $\phi_{21}^{\mathrm{CO}}$ (solid curve) for ${ }^{12} \mathrm{CO}(J=2 \rightarrow 1)$ in Orion $\mathrm{KL}$ (using the left vertical scale) for a case where the angle $\theta$ between the incident linear polarization and the magnetic field orientation varies linearly with frequency (or velocity) at a rate of $d \theta / d v=-1 \mathrm{deg} /\left(\mathrm{km} \mathrm{s}^{-1}\right.$ ) (with $\theta=0$ at $v=0$ ). The underlying linear polarization radiation profile $u(v)$, shown with the dashed curve, has been normalized in the figure but has a peak antenna temperature of $1 \mathrm{~K}$. The corresponding Stokes $V$ spectrum is also shown (dot-dashed curve), using the vertical scale on the right. This spectrum is proportional to $-2 \sin (\theta) \cos (\theta) u(v) \sin \left(\phi_{21}^{\mathrm{CO}}\right)$ (see Equation (5)). Note that the Stokes $V$ spectrum now has a symmetric profile about $v=0$. The magnetic field strength was set to $0.1 \mathrm{mG}$.

levels found in maser transitions that require unreasonably large magnetic field strengths when interpreted within the context of the Zeeman effect (Watson 2009). Furthermore, our model is also consistent with other observations of $\mathrm{SiO}$ maser lines in evolved stars that showed a correlation between measured levels of linear and circular polarizations, no correlation between levels of circular polarization and Stokes $I$, and a case where circular polarization is more important than linear polarization (Herpin et al. 2006).

We stress, however, that although some $\mathrm{SiO}$ maser Stokes $V$ line profiles presented in Cotton et al. (2011) match well the asymmetric profile resulting from our simplest calculations shown in Figures 5 and 6 (i.e., when the angle $\theta$ between the incident linear polarization and the magnetic field orientation is constant across the spectrum), many of their spectra show (quasi-)symmetric Stokes $V$ profiles that are different from anything we presented. More precisely, if the ${ }^{12} \mathrm{CO}(J=2 \rightarrow 1)$ line in Orion KL is expected to yield little to no polarization at or near the center of the line, the same cannot apply to maser lines in view of the nature of the stimulated emission process. That is, several of the Cotton et al. (2011) Stokes $V$ spectra show a maximum (in the absolute sense) near the center of the line, not a near-zero value. It follows that the symmetric Stokes $V$ spectrum of Figure 7 cannot, by itself, explain the aforementioned results of Cotton et al. (2011). It is still possible, however, that the presence of several maser spots within the telescope beam could lead to such (quasi-)symmetric line profiles under the assumption that the underlying Stokes $V$ spectrum of a given maser is as shown in Figure 7 and the masers' systemic velocities are spread across the extent of the observed spectral line. The combination of these relatively shifted Stokes $V$ spectra (i.e., as in Figure 7) could thus yield something akin to the (quasi-)symmetric line profile of Cotton et al. (2011). However, it is perhaps safer at this point to await the results of an analysis similar to the one presented in this paper, but specifically tailored to maser emission, to find out if our resonant scattering model can account for all aspects of $\mathrm{SiO}$ maser circular polarization spectra with a minimum number of assumptions. This we intend to attempt in a future publication.

Our results also have implications for Zeeman measurements in non-masing environments (e.g., in molecular clouds). For example, it is clear from our discussion of Section 4.3.2 that $\mathrm{HNCO}$ is a Zeeman sensitive molecule (in contrast to $\mathrm{CO}$ and $\mathrm{HCN}$ ). However, it is likely that our observations of Figure 2 would be dismissed as due to instrumental artifacts when analyzed within the context of the Zeeman effect (e.g., leakage from Stokes $I$ to Stokes $V$ ). But when studied in conjunction with the $\mathrm{HCN}$ result presented in the same spectrum and that for ${ }^{12} \mathrm{CO}(J=2 \rightarrow 1)$ shown in Figure 1, another interpretation is warranted.

\section{CONCLUSION}

We presented measurements of circular polarization from rotational spectral lines of molecular species in Orion KL obtained at the Caltech Submillimeter Observatory with the Four-Stokes-Parameter Spectral Line Polarimeter. We measured levels of polarization of up to $1 \%-2 \%$ for ${ }^{12} \mathrm{CO}(J=2 \rightarrow 1)$ and $\mathrm{HNCO} / \mathrm{HN}^{13} \mathrm{CO}\left(N_{K_{a} K_{c}}=12_{1,12} \rightarrow 11_{1,11}\right)$, while none was detected for $\mathrm{HCN}(J=3 \rightarrow 2)$. We further presented a physical model based on resonant scattering in an attempt to explain our observations, through the conversion of linear polarization to circular polarization. We also showed that this effect is proportional to the square of the magnitude of the plane of the sky component of the magnetic field and therefore opens up the possibility of measuring this parameter from circular polarization measurements of Zeeman insensitive molecules. We intend to study this in an upcoming paper.

M.H. is grateful to C. Cohen-Tannoudji of the Ecole Normale Supérieure de Paris for an insightful discussion. M.H.'s research is funded through the NSERC Discovery Grant, Canada Research Chair, and Western's Academic Development Fund programs. T.H. is funded by the Alexander von Humboldt foundation in Germany. The Caltech Submillimeter Observatory is operated by the California Institute of Technology under cooperative agreement with the National Science Foundation (AST-0838261).

\section{REFERENCES}

Attard, M., Houde, M., Novak, G., et al. 2009, ApJ, 702, 1584

Blake, G. A., Sutton, E. C., Masson, C. R., \& Phillips, T. G. 1987, ApJ, 315,621

Brogan, C. L., \& Troland, T. H. 2001, ApJ, 560, 821

Chandrasekhar, S., \& Fermi, E. 1953, ApJ, 118, 113

Chitsazzadeh, S., Houde, M., Hildebrand, R. H., \& Vaillancourt, J. E. 2012, ApJ, 749,45

Cohen-Tannoudji, C., Diu, B., \& Laloë, F. 1977, Mécanique Quantique I (Paris: Hermann)

Cohen-Tannoudji, C., Dupont-Roc, J., \& Grynberg, G. 1988, Processus d'Interaction entre Photons et Atomes (Paris: CNRS)

Cortes, P. C., Crutcher, R. M., \& Watson, W. D. 2005, ApJ, 628, 780

Cotton, W. D., Ragland, S., \& Danchi, W. C. 2011, ApJ, 736, 96

Crutcher, R. M., Troland, T. H., Lazareff, B., Paubert, G., \& Kazès, I. 1999, ApJL, 514, 121

Davis, R. E., \& Muenter, J. S. 1974, JChPh, 61, 2940 
Decin, L., De Beck, E., Brüuken, S., et al. 2012, A\&A, 516, 69

Deguchi, S., \& Watson, W. D. 1985, ApJ, 289, 621

Dotson, J. L., Vaillancourt, J. E., Kirby, L., et al. 2010, ApJS, 186, 406

Falceta-Gonçalves, D., Lazarian, A., \& Houde, M. 2010, ApJ, 713, 1376

Falgarone, E., Troland, T. H., Crutcher, R. M., \& Paubert, G. 2008, A\&A, 487,247

Girart, J. M., Greaves, J. S., Crutcher, R. M., \& Lai, S.-P. 2004, Ap\&SS, 292, 119

Girart, J. M., Rao, R., \& Marrone, D. P. 2006, Sci, 313, 812

Glenn, J., Walker, C. K., Bieging, J. H., \& Jewell, P. R. 1997, ApJL, 487, 89

Goldreich, P., \& Kylafis, N. D. 1981, ApJL, 243, 75

Gordy, W., \& Cook, B. L. 1984, Microwave Molecular Spectroscopy (3rd ed.; New York: Wiley)

Grynberg, G., Aspect, A., \& Fabre, C. 2010, Introduction to Quantum Optics (Cambridge: Cambridge Univ. Press)

Heiles, C. 1997, ApJS, 111, 245

Heiles, C. 2000, AJ, 119, 923

Herpin, F., Baudry, A., Thum, C., Morris, D., \& Wiesemeyer, H. 2006, A\&A, 450,667

Heyer, M., Gong, H., Ostriker, E., \& Brunt, C. 2008, ApJ, 680, 420

Hezareh, T., \& Houde, H. 2010, PASP, 122, 786

Hildebrand, R. H., Kirby, L., Dotson, J. L., Houde, M., \& Vaillancourt, J. E. 2009, ApJ, 696, 567

Houde, M. 2004, ApJL, 616, 111

Houde, M., Bastien, P., Peng, R., Phillips, T. G., \& Yoshida, H. 2000a, ApJ, 536,847

Houde, M., Peng, R., Phillips, T. G., Bastien, P., \& Yoshida, H. 2000b, ApJ, 537,245
Houde, M., Phillips, T. G., Bastien, P., Peng, R., \& Yoshida, H. 2001, ApJ, 547,311

Houde, M., Rao, R., Vaillancourt, J. E., \& Hildebrand, R. H. 2011, ApJ, 733, 109

Houde, M., Vaillancourt, J. E., Hildebrand, R. H., Chitsazzadeh, S., \& Kirby, L. 2009, ApJ, 706, 1504

Li, H., Attard, M., Dowell, C. D., et al. 2006, Proc. SPIE, 6275, 48

Li, H., \& Houde, M. 2008, ApJ, 677, 1151

Matthews, B. C., McPhee, C., Fissel, L., \& Curran, R. L. 2009, ApJS, 182,143

Muñoz, D. J., Marrone, D. P., Moran, J. M., \& Rao, R. 2012, ApJ, 745,115

Novak, G., et al. 2004, Proc. SPIE, 5498, 278

Pickett, H. M., Poynter, R. L., Cohen, E. A., et al. 1998, JQSRT, 60, 883

Plume, R., Bergin, E. A., Phillips, T. G., et al. 2012, ApJ, 744, 28

Rybicki, G. B., \& Lightman, A. P. 1979, Radiative Processes in Astrophysics (New York: Wiley)

Schilke, P., Bendford, D. J., Hunter, T. R., Lis, D. C., \& Phillips, T. G. 2001, ApJS, 132, 281

Shull, J. M., \& Draine, B. T. 1990, in Interstellar Processes, ed. D. J. Hollenback \& H. A. Thronson, Jr. (Dordrecht: Kluwer), 225

Tideswell, D. M., Fuller, G. A., Millar, T. J., \& Markwick, A. J. 2010, A\&A, 510, A85

Tilley, D. A., \& Balsara, D. S. 2010, MNRAS, 406, 1201

Tilley, D. A., \& Balsara, D. S. 2011, MNRAS, 415, 368

Vaillancourt, J. E., \& Matthews, B. C. 2012, ApJS, 201, 13

Watson, W. D. 2009, RMxAA, 36, 113

Wiebe, D. S., \& Watson, W. D. 1998, ApJL, 503, 71 